\title{
Causal Anomalies in Kaluza-Klein Gravity Theories
}

\author{
M.J. Rebouças* and A.F.F Teixeira ${ }^{\dagger}$ \\ Centro Brasileiro de Pesquisas Físicas \\ Departamento de Relatividade e Partículas \\ Rua Dr. Xavier Sigaud 150 \\ 22290-180 Rio de Janeiro - RJ, Brazil
}

October 11, 2018

\begin{abstract}
Causal anomalies in two Kaluza-Klein gravity theories are examined, particularly as to whether these theories permit solutions in which the causality principle is violated. It is found that similarly to general relativity the field equations of the space-time-mass Kaluza-Klein (STM-KK) gravity theory do not exclude violation of causality of Gödel type, whereas the induced matter Kaluza-Klein (IM-KK) gravity rules out noncausal Gödel-type models. The induced matter version of general relativity is shown to be an efficient therapy for causal anomalies that occurs in a wide class of noncausal geometries. Perfect fluid and dust Gödel-type solutions of the STM-KK field equations are studied. It is shown that every Gödel-type perfect fluid solution is isometric to the unique dust solution of the STM-KK field equations. The question as to whether 5-D Gödel-type non-causal geometries induce any physically acceptable 4-D energy-momentum tensor is also addressed.
\end{abstract}

*INTERNET: REBOUCAS@CAT.CBPF.BR

†INTERNET:TEIXEIRA@NOVELL.CAT.CBPF.BR 


\section{Introduction}

Kaluza-Klein-type theories in five or more dimensions have been of interest in a few contexts. In the framework of gauge theories they have been used in the quest for unification of the fundamental interactions in physics. The idea that the various interactions in nature might be unified by enlarging the dimensionality of the space-time has a long history that goes back to the works of Kaluza and Klein [1] - [2]. They showed how one could unify Einstein's theory of gravitation and Maxwell's theory of electromagnetism in a five-dimensional framework. The higher-dimensional Kaluza-Klein approach to unification was later used by the unified-field theorists, especially among those investigating the eleven-dimensional supergravity and ten-dimensional superstrings.

In 1983, Wesson [3, 4] has given an impetus to the study of Kaluza-Klein type theories by investigating a five-dimensional Kaluza-Klein theory of gravitation with a variable rest mass. In this theory we have five-dimensional (5-D) space-time-mass manifolds, and the fifth dimension is a convenient mathematical way of geometrizing the rest mass and of allowing one to study the possibility that it may be variable. The four-dimensional (4-D) general relativity theory is recovered when the rate of change of the rest mass is zero. We shall refer to this theory as space-time-mass Kaluza-Klein gravity theory (STM-KK gravity theory, for short). Ever since the foundations of the STM-KK gravity theory were laid, there have been investigations on its potentialities, particularly as concerns to its consequences for astrophysics and cosmology, the confrontation between theory and observation, its consistency with Mach's principle, the solutions to its field equations, and the like [4] - [18]. For fair list of references on the STM-KK theory see Overduin and Wesson 19$]$.

More recently, Wesson and co-workers [5, 20] have introduced a new approach to general relativity, conceptually in line with an old idea due to Einstein [21], in which the matter and its role in the determination of the space-time geometry is given from a purely five-dimensional geometrical point of view. In their 5-D version of general relativity the field equations are given by

$$
\widehat{G}_{A B}=0
$$

Henceforth, the five-dimensional geometrical objects are denoted by overhats and Latin 
letters are 5-D indices and run from 0 to 4. The 5-D vacuum field equations (1.1) give rise to both curvature and matter in 4-D. Indeed, it can be shown [20] that it is always possible to rewrite the field equations (1.1) as a set of fifteen equations, ten of which are precisely Einstein's field equations

$$
G_{\alpha \beta}=\kappa T_{\alpha \beta}
$$

in 4-D with an induced energy-momentum

$$
\begin{aligned}
T_{\alpha \beta}= & \frac{\phi_{\alpha ; \beta}}{\phi}-\frac{\varepsilon}{2 \phi^{2}}\left\{\frac{\phi^{*} g_{\alpha \beta}^{*}}{\phi}-g_{\alpha \beta}^{* *}+g^{\gamma \delta} g_{\alpha \gamma}^{*} g_{\beta \delta}^{*}-\frac{g^{\gamma \delta} g_{\gamma \delta}^{*} g_{\alpha \beta}^{*}}{2}\right. \\
& \left.+\frac{g_{\alpha \beta}}{4}\left[g^{* \gamma \delta} g_{\gamma \delta}^{*}+\left(g^{\gamma \delta} g_{\gamma \delta}^{*}\right)^{2}\right]\right\}
\end{aligned}
$$

where from now on Greek letters denote 4 -D indices and run from 0 to $3, g_{44} \equiv \varepsilon \phi$ with $\varepsilon= \pm 1, \phi_{\alpha} \equiv \partial \phi / \partial x^{\alpha}$, a star denotes $\partial / \partial x^{4}$, and a semicolon denotes the usual 4-D covariant derivative. Obviously, the remaining five equations (a wave equation and four conservation laws) are automatically satisfied by any solution of the 5-D vacuum equations (1.1). Thus, not only the matter but also its role in the determination of the geometry of the 4-D space-time can be considered to have a five-dimensional geometrical origin. This is an elegant step towards the realization of the Einstein's vision [21] - [23] of nature as pure geometry in that it unifies the gravitational field with its source (not just with other fields) within a purely 5-D geometrical framework. We shall refer to this 5-D version of general relativity as induced matter Kaluza-Klein gravity theory (IM-KK gravity theory, for short). The IM-KK theory has become a focus of a recent research field [19]. The basic features of the theory have been explored by Wesson and others [24 [28], whereas the implications for cosmology and astrophysics have been investigated by a number of researchers [29] - 49]. For an updated list of references on IM-KK theory and related matters we again refer the reader to Overduin and Wesson [19].

It has long been known that there are solutions to the Einstein field equations which possess causal anomalies in the form of closed time-like curves. The famous solution found by Gödel [50] in 1949 might not be the first but it certainly is the best known example of a cosmological model which makes it apparent that the general relativity, as it is normally formulated, does not exclude the existence of closed time-like world lines, despite its Lorentzian character which leads to the local validity of the causality 
principle. The Gödel model is a solution of Einstein's field equations with cosmological constant for incoherent matter (dust) with rotation. Owing to its striking properties Gödel's model has a well-recognized importance and has to a certain extent motivated the investigations on rotating cosmological Gödel-type models and on causal anomalies in the framework of general relativity [51 - 66] and other theories of gravitation [67] [75]. Among the relevant results on Gödel-type models in general relativity, it is worth mentioning the Bampi-Zordan theorem [53], which states that every Gödel-type solution of the 4-D Einstein's equations whose energy-momentum tensor is that of a perfect fluid is necessarily isometric to the Gödel model [50].

In this work we shall examine the causal anomalies of Gödel-type in the two abovementioned Kaluza-Klein gravity theories. We shall be particularly concerned with the question as to whether these theories of gravitation allow solutions in which the causality is violated. To this end, we investigate whether these theories admit the 5-D Gödeltype metrics [76] as solutions to their field equations. Perfect fluid and dust Gödel-type solutions of the STM-KK field equations (with and without cosmological constant $\Lambda$ ) are discussed. We also extend the Bampi-Zordan results [53 to the context of STM-KK theory, i.e., we show that every Gödel-type perfect fluid solution of the STM-KK field equations is isometric to the 5-D Gödel's geometry, which is shown to be the only Gödeltype dust solution of the STM-KK theory. There emerges from our results that, similarly to the general relativity theory, the STM-KK theory [3] - [4] does not exclude violation of causality of the Gödel type.

In the context of the induced matter theory of gravitation, besides examining possible Gödel-type solutions of the IM-KK field equations, we discuss the question as to whether 5-D Gödel-type non-causal geometries discussed in Ref. [76] induce any physically acceptable 4-D energy-momentum tensor. We show that there is no curved Gödel-type metric which is solution to the IM-KK field equations (1.1), making apparent that the non-causal Gödel-type 5-D curved geometries cannot induce any type of matter in 4-D. Although one cannot yet recommend the 5-D induced matter version of general relativity [5, 20] as an efficient therapy for all sort of causal pathologies that occur in general relativity, as it is normally formulated, our results are certainly an important step towards this conjecture in that they prove the effectiveness of the induced matter theory as a therapy for causal 
anomalies of the 5-D Gödel-type family of metrics.

\section{Solutions and Causal Anomalies}

In this section we shall first be concerned with Gödel-type solutions of both Kaluza-Klein theories of gravitation we have discussed in the Introduction. To this end we write the 5-D Gödel-type line element in the form

$$
d \hat{s}^{2}=[d t+H(r) d \Phi]^{2}-D^{2}(r) d \Phi^{2}-d r^{2}-d z^{2}-d \psi^{2}
$$

where $H(r)$ and $D(r)$ are arbitrary real functions of $r$, and the coordinates are such that $x^{0}=t$ (time), $x^{1,2,3}=r, \Phi, z$ (spacelike cylindrical coordinates) and $x^{4}=\psi$ (extra coordinate).

Defining the one-forms $\widehat{\Theta}^{A}$ according to

$$
\widehat{\Theta}^{0}=d t+H(r) d \Phi, \quad \widehat{\Theta}^{1}=d r, \quad \widehat{\Theta}^{2}=D(r) d \Phi, \quad \widehat{\Theta}^{3}=d z, \quad \widehat{\Theta}^{4}=d \psi,
$$

the Gödel-type line element (2.1) can be written as

$$
d \hat{s}^{2}=\widehat{\eta}_{A B} \widehat{\Theta}^{A} \widehat{\Theta}^{B}=\left(\widehat{\Theta}^{0}\right)^{2}-\left(\widehat{\Theta}^{1}\right)^{2}-\left(\widehat{\Theta}^{2}\right)^{2}-\left(\widehat{\Theta}^{3}\right)^{2}-\left(\widehat{\Theta}^{4}\right)^{2},
$$

where here and in what follows capital Latin letters are frame indices and run from 0 to 4;

they are raised and lowered with Lorentz matrices $\widehat{\eta}^{A B}=\widehat{\eta}_{A B}=\operatorname{diag}(+1,-1,-1,-1,-1)$, respectively.

A straightforward calculation, performed by using the computer algebra package CLASSI [77, 78], gives the frame components of the Einstein tensor $\widehat{G}_{A B}=\widehat{R}_{A B}-\frac{1}{2} R \widehat{\eta}_{A B}$ in the frame (2.2). We find that the nonvanishing components are

$$
\begin{aligned}
\widehat{G}_{00} & =-\frac{D^{\prime \prime}}{D}+\frac{3}{4}\left(\frac{H^{\prime}}{D}\right)^{2}, \\
\widehat{G}_{02} & =\frac{1}{2}\left(\frac{H^{\prime}}{D}\right)^{\prime}, \\
\widehat{G}_{11} & =\widehat{G}_{22}=\frac{1}{4}\left(\frac{H^{\prime}}{D}\right)^{2}, \\
\widehat{G}_{33} & =\widehat{G}_{44}=\frac{D^{\prime \prime}}{D}-\frac{1}{4}\left(\frac{H^{\prime}}{D}\right)^{2},
\end{aligned}
$$


where the prime denotes derivative with respect to $r$.

The field equations in the STM-KK gravity theory are taken to be 5-D analogues of the usual 4-D Einstein's field equations 44, 5], namely

$$
\widehat{G}_{A B}+\Lambda \widehat{\eta}_{A B}=\kappa \widehat{T}_{A B}
$$

where $\Lambda$ and $\kappa$ are the cosmological and the Einstein constants, respectively. We shall use units such that $\kappa=c=1$, where $c$ denotes the speed of light. We shall take the 5-D energy-momentum tensor $\widehat{T}_{A B}$ to be that of a comoving perfect fluid [4, 12], viz.,

$$
\widehat{T}_{A B}=\operatorname{diag}\left(\rho, p, p, p, p_{4}\right)
$$

wherein $\rho$ is the matter density, $p$ is the isotropic pressure and $p_{4}$ denotes the pressure along the extra direction defined by $\widehat{\Theta}^{4}=d \psi$.

When $\Lambda=0$, the field equations (2.8) and the energy-momentum tensor (2.9) require that $\widehat{G}_{02}=0$, which in turn implies

$$
\frac{H^{\prime}}{D}=\text { const } \equiv-2 \omega
$$

From equations (2.4) - (2.7) and (2.10) one finds that the remaining field equations reduce to

$$
\begin{aligned}
\rho & =3 \omega^{2}-\frac{D^{\prime \prime}}{D} \\
p & =\omega^{2}, \\
p_{4} & =p=\frac{D^{\prime \prime}}{D}-\omega^{2} .
\end{aligned}
$$

The equations (2.12) and (2.13) then lead to

$$
\frac{D^{\prime \prime}}{D}=\text { const } \equiv m^{2}
$$

where for the present solution $m^{2}=2 \omega^{2}$.

Now, taking into account the irreducible set of isometrically nonequivalent homogeneous Gödel-type metrics [76], the solution to field equations (2.10) and (2.14) can always be brought into the form $(2.1)$ with 


$$
\begin{aligned}
& D(r)=\frac{1}{m} \sinh (m r), \\
& H(r)=\frac{\sqrt{2}}{m}[1-\cosh (m r)],
\end{aligned}
$$

where, without loss of generality, we have used $m=\sqrt{2} \omega$ for definiteness.

From (2.11) - (2.13) one obtains the equation of state

$$
p=\rho=p_{4}=\omega^{2},
$$

which characterizes a stiff matter type of fluid. In (2.16) the positivity of the density and of the pressure are manifestly fulfilled.

Solutions with nonvanishing cosmological constant $\Lambda$ can be similarly found. Indeed, here again $\widehat{G}_{02}=0$ implies (2.10), and the remaining STM-KK field equations reduce to

$$
\begin{aligned}
\widehat{G}_{00} & =-\frac{D^{\prime \prime}}{D}+3 \omega^{2}=\rho-\Lambda, \\
\widehat{G}_{11} & =\widehat{G}_{22}=\omega^{2}=p+\Lambda, \\
\widehat{G}_{33} & =\frac{D^{\prime \prime}}{D}-\omega^{2}=p+\Lambda, \\
\widehat{G}_{44} & =\frac{D^{\prime \prime}}{D}-\omega^{2}=p_{4}+\Lambda .
\end{aligned}
$$

On the other hand, it is straightforward to show that equations (2.18) - (2.19) lead to (2.14), which together with (2.10) gives rise to the solution (2.15); but now using (2.17), (2.19), (2.20) and (2.14), instead of the equation of state (2.16) one finds

$$
\begin{aligned}
& \rho=\omega^{2}+\Lambda, \\
& p=\omega^{2}-\Lambda=p_{4},
\end{aligned}
$$

which does not correspond to a stiff matter type of fluid unless $\Lambda=0$. Here the positivity of the density $\rho$ and the pressure $p$ are ensured provided that the cosmological constant lies in the range $-\omega^{2} \leq \Lambda \leq \omega^{2}$. In the case where $0 \leq \Lambda \leq \omega^{2}$ we have a linear barotropic equation of state, viz.,

$$
p=\gamma \rho, \quad 0 \leq \gamma \leq 1,
$$

where $\gamma \equiv\left(\omega^{2}-\Lambda\right) /\left(\omega^{2}+\Lambda\right)$. Clearly the limiting case when $\Lambda=0$ is the previous solution given by (2.1), (2.15) and (2.16). 
There also exists a Gödel-type dust solution of STM-KK field equations. Indeed, the solution is again given by (2.15) but now, using (2.21) and (2.22) one obtains

$$
\rho=2 \Lambda=2 \omega^{2}>0
$$

It should be stressed that the solution (2.15) with the density given by (2.24) is the only Gödel-type dust solution of the STM-KK field equations. We shall hereafter refer to this solution as the 5-D Gödel model.

We shall now show how the above results give rise to the extension of the Bampi-Zordan theorem [53] we have referred to in the Introduction. Indeed, the equations (2.10) and (2.14) hold for all the above solutions of the STM-KK theory. But for arbitrary constants $m^{2}$ and $\omega$, equations (2.10) and (2.14) are the necessary and sufficient conditions for a 5-D Gödel-type manifold to be (locally) homogeneous [76]. Therefore, taking also into account the theorem 2 of Ref. [76], the above solutions are (locally) homogeneous and admit a group of isometry $G_{r}$ of dimension $r=7$. Moreover, as there is a fixed relation $\left(m^{2}=2 \omega^{2}\right)$ between the essential parameters $m^{2}$ and $\omega$ for them all, they characterize (locally) just one 5-D STM manifold [76], which is nothing but the 5-D counterpart of the 4-D Gödel (dust) model. Thus, all perfect fluid Gödel-type solutions of STM-KK field equations (2.8) are isometric to the 5-D Gödel metric (2.1) and (2.15), which is the only Gödel-type dust solution of the STM-KK theory.

The 5-D Gödel metric (2.1) with $H$ and $D$ given by (2.15) permits violation of causality. Indeed, the line element (2.1) can be rewritten as

$$
d \hat{s}^{2}=d t^{2}+2 H(r) d t d \Phi-d r^{2}-G(r) d \Phi^{2}-d z^{2}-d \psi^{2}
$$

where $G(r)=D^{2}-H^{2}$. In this form it is clear that the existence of closed timelike curves depends on the behaviour of $G(r)$. If $G(r)<0$ within a certain range of $r\left(r_{1}<r<r_{2}\right.$, say), Gödel's circles defined by $t, z, \psi, r=$ const are closed timelike curves. Particularly, for the above 5-D Gödel geometry given by (2.15) and (2.25), it is easy to find out that there is a critical radius $r_{c}$, defined by $\sinh \left(m r_{c} / 2\right)=1$, such that for all $r_{c}<r<\infty$ the circles $t, z, \psi=$ const and $r=$ const $>r_{c}$ are closed timelike curves. Thus, similarly to the general relativity theory, the STM-KK gravity theory admits solutions which allow the violation of causality. 
Following the reasoning outlined in the previous paragraph one can show [76 that there are closed timelike curves for all classes of homogeneous 5-D manifolds endowed with a Gödel-type metric (2.1) [or equivalently (2.25)]. However, in what follows we shall show that these types of non-causal curved manifolds cannot be accomodated in the context of the induced matter Kaluza-Klein gravity theory [5, 20].

It should be noticed from the outset that since $\phi=1$ and the metric components of (2.1) do not depend on the fifth coordinate $\psi$, all components of the induced energy momentum-tensor (1.3) obviously vanish, i.e., the 5-D Gödel-type metrics (2.1) induce no matter in 4-D. As a matter of fact, there is no curved manifold with 5-D metric (2.1) solution to the field equations (1.1). To show this, we first note that as $\widehat{G}_{02}=0$ then (2.10) holds again. On the other hand, taking in account (2.4 - (2.7) and (2.10) one easily finds that the IM-KK field equations (1.1) are fulfilled if and only if $m^{2}=\omega=0$, which leads to

$$
H=a \quad \text { and } \quad D=b r+c,
$$

where $a, b$ and $c$ are arbitrary real constants. However, the constants $a, b$ and $c$ have no physical meaning, and can be taken to be $a=c=0$ and $b=1$ by a suitable choice of coordinates. Indeed, if one performs the coordinate transformations

$$
\begin{aligned}
t & =\bar{t}-\frac{a}{b} \bar{\Phi}, \quad r=\bar{r}-\frac{c}{b}, \\
\Phi & =\frac{\bar{\Phi}}{b}, \quad z=\bar{z}, \quad \psi=\bar{\psi},
\end{aligned}
$$

the line element (2.25) becomes

$$
d \hat{s}^{2}=d \bar{t}^{2}-d \bar{r}^{2}-\bar{r}^{2} d \bar{\Phi}^{2}-d \bar{z}^{2}-d \bar{\psi}^{2}
$$

in which we obviously have $G(\bar{r})=\bar{r}^{2}>0$ for $\bar{r} \neq 0$. The line element (2.29) corresponds to a manifestly flat 5-D manifold, making it clear that the underlying manifold can be taken to be the simply connected Euclidean manifold $\mathbb{R}^{5}$, and therefore as $G(\bar{r})>0$ no closed time-like circles are permitted. Furthermore the above results clearly show that the IM-KK theory does not admit any curved 5-D Gödel-type metric (2.1) as solution to its field equations (1.1).

However, in a recent work McManus [34] has shown that a one-parameter family of solutions of the field equations (1.1) previously found by Ponce de Leon [10] (see also 
Wesson [30, 28] and Coley et al. [36]) was in fact flat in five dimensions, i.e., the 5-D Riemann tensor vanishes identically. And yet the corresponding 4-D induced models were shown to be curved. Actually, the induced 4-D space-times studied in [10] constitute a perfect fluid family of Friedmann-Robertson-Walker models. A question which naturally arises here is whether the above 5-D flat solutions of the IM-KK field equations can similarly give rise to any 4-D curved space-time. By using the eqs. (5.3) of Ref. [58 or simply by using a computer algebra package as, e.g., CLASSI [77, 78] it is straightforward to show that when $m^{2}=\omega=0$ the above 5 -D flat geometry [with $m^{2}$ and $\omega$ defined by (2.10) and (2.14)] gives rise to nothing but the 4-D Minkowski (flat) space-time.

\section{Concluding Remarks}

In general relativity, the causal structure of 4-D space-time has locally the same qualitative nature as the flat space-time of special relativity - causality holds locally. The global question, however, is left open and significant differences can occur. On large scale, the violation of causality is not excluded. A few familiar space-times make it clear that general relativity, as it is normally formulated, does not phohibit closed time-like curves. The Gödel model [50] is perhaps the best known example of a cosmological solution of Einstein's equations in which causality may be violated.

In a recent article the main properties of the five-dimensional Riemannian manifolds endowed with a 5-D analogue of the 4-D Gödel-type metric were investigated and the main mathematical properties discussed [76]. Among several results, an irreducible set of isometrically nonequivalent 5D (locally) homogeneous Gödel-type metrics were exhibited. It was also shown that, apart from the degenerated Gödel-type metric, for which the

rotation $\Omega^{\mu}=\left(0,0,0, \frac{H^{\prime}}{2 D}\right)$ vanishes, in all classes of homogeneous Gödel-type geometries there is breakdown of causality. As no use of any particular field equations was made, these results hold for any 5-D Gödel-type manifolds regardless of the underlying 5-D Kaluza-Klein theory of gravitation.

In this work we have investigated the 5-D Gödel-type geometries from a more physical perspective. Particularly, we have examined the question as to whether the spacetime-mass and the induced mass theories of gravitation (STM-KK and IM-KK gravity 
theories [3] - [5], 20]) allow solutions that violated causality. We have shown that, similarly to general relativity, the STM-KK theory permits noncausal solutions of Gödel type. However, the IM-KK gravity theory is shown to exclude this type of noncausal geometries.

Two perfect fluid and a dust solutions of the STM-KK gravity theory have been found. Actually, we have extended to the context of the STM-KK theory the BampiZordan results [53], proving that all Gödel-type perfect-fluid solutions of the STM-KK field equations are isometric to the unique Gödel-type dust solution of this theory.

Regarding the question as to whether the 5-D Gödel-type noncausal geometries [76] induce any physically acceptable 4-D matter, clearly since $\phi=1$ and the metric components of (2.1) do not depend on the fifth coordinate $\psi$, this noncausal family of geometries induce no 4-D matter. In agreement with this fact we have shown that no curved solution of Gödel-type is permited in the IM-KK gravity theory. Moreover the surviving flat solution of the IM-KK theory is shown to give rise to the 4-D Minkowski space-time.

We remark that the 5-D Gödel-type metric we have studied is the simplest stationary 5-D class of geometries for which $\psi=$ const section is the 4-D Gödel-type metric of general relativity. Nevertheless, the 5-D seed metric (2.1) does share the most important features of the 4-D Gödel-type counterpart, namely it permits violation of causality, admits nonzero rotation $\Omega^{\mu}$, and has the most relevant symmetries $\left(\partial_{t}, \partial_{\phi}\right.$ and $\partial_{z}$ are Killing vector fields). However, as (2.1) does not depend on the fifth coordinate $\psi$ a radiation-like equation of state is an underlying assumption of this work regarding the IM-KK theory. The dependence of the 5-D metric on the extra coordinate gives to the 5-D IM-KK field equations (1.1) a rich enough structure which permits that matter of a very general type can be induced in 4-D. An exhaustive study of causal anomalies and solutions in a more general 5-D geometrical setting has been carried out and we hope to publish an account of our results shortly elsewhere. We anticipate, however, that if one demands that the 5-D seed generalized Gödel-type metrics share the above-mentioned properties of the 4-D Gödel-type counterpart, it can be shown that the IM-KK theory also excludes a large class of noncausal generalized Gödel-type stationary geometries with two arbitrary functions $F(\psi)$ and $G(\psi)$ of the fifth coordinate $\psi$, besides, obviously, the functions $H(r)$ and $D(r)$.

Although one cannot yet recommend the 5-D induced matter Wesson's version of 
general relativity [5, 20] as an efficient treatment for all sort of causal anomalies in general

relativity, our results constitute an important step in this direction inasmuch as they make apparent the effectiveness of the induced matter theory as a therapy for causal anomalies of the 5-D Gödel-type families of metrics discussed in this work.

\section{Acknowledgement}

The authors gratefully acknowledge financial assistance from CNPq.

\section{References}

[1] Th. Kaluza, Sitzungsberichte preuss. Akad. Wiss. - Phys. Math. Klasse LIV, 966 (1921).

[2] O. Z. Klein, Phys. Z. 37, 895 (1926).

[3] P. S. Wesson, Astron. Astrophys. 119, 145 (1983).

[4] P. S. Wesson, Gen. Rel. Grav. 16, 193 (1984).

[5] P. S. Wesson, Gen. Rel. Grav. 22, 707 (1990).

[6] P. S. Wesson, Astron. Astrophys. 166, 1 (1986).

[7] P. S. Wesson, Astron. Astrophys. 189, 4 (1988).

[8] S. Chatterjee, Gen. Rel. Grav. 18, 1073 (1986).

[9] S. Chatterjee, Astron. Astrophys. 230, 1 (1990).

[10] J. Ponce de Leon, Gen. Rel. Grav. 20, 539 (1988).

[11] Ø. Grøn and H. H. Soleng, Gen. Rel. Grav. 20, 1115 (1988).

[12] Ø. Grøn, Astron. Astrophys. 193, 1 (1988).

[13] G.-W. Ma, Phys. Lett. A 143, 183 (1990). 
[14] G.-W. Ma, Phys. Lett. A 146, 375 (1990).

[15] A. A. Coley, Astron. Astrophys. 233, 305 (1990).

[16] L. K. Chi, Gen. Rel. Grav. 22, 1347 (1990).

[17] T. Fukui, Gen. Rel. Grav. 19, 43 (1987).

[18] T. Fukui, Astron. Astrophys. 253, 1 (1992).

[19] J. M. Overduin and P. S. Wesson, Phys. Rep. 283, 303 (1997).

[20] P. S. Wesson and J. Ponce de Leon, J. Math. Phys. 33, 3883 (1992).

[21] A. Einstein, The meaning of general relativity, p. 129. Princeton U. P., Princeton (1956).

[22] J. A. Wheeler, Einstein's vision, Springer-Verlag, Berlin (1968).

[23] A. Salam, Rev. Mod. Phys. 52, 525 (1980).

[24] J. Ponce de Leon and P. S. Wesson, J. Math. Phys. 34, 4080 (1993).

[25] P. S. Wesson, J. Ponce de Leon, P. H. Lim and H. Liu, Int. J. Mod. Phys. D 2, 163 (1993).

[26] P. S. Wesson, Mod. Phys. Lett. A 10, 15 (1995).

[27] S. Rippl, C. Romero and R. Tavakol, Class. Quant. Grav. 12, 2411 (1995).

[28] P. S. Wesson, J. Ponce de Leon, H. Liu, B. Mashhoon, D. Kalligas, C. W. F. Everitt, A. Billyard, P. H. Lim and J. M. Overduin, Int. J. Mod. Phys. A 11, 3247 (1996).

[29] P. S. Wesson, Mod. Phys. Lett. A 7, 921 (1992).

[30] P. S. Wesson, Astrophys. J. 394, 19 (1992).

[31] P. S. Wesson and H. Liu, Astrophys. J. 440, 1 (1995).

[32] P. S. Wesson and J. Ponce de Leon, Astron. Astrophys. 294, 1 (1995). 
[33] H. Liu and P. S. Wesson, Int. J. Mod. Phys. D 3, 627 (1994).

[34] D. J. McManus, J. Math. Phys. 35, 4889 (1994).

[35] A. A. Coley, Astrophys. J. 427, 585 (1994).

[36] A. A. Coley and D. J. McManus, J. Math. Phys. 36, 335 (1995).

[37] P. S. Wesson and J. Ponce de Leon, Gen. Rel. Grav. 26, 555 (1994).

[38] B. Mashhoon, H. Liu and P. S. Wesson, Phys. Lett. B 331, 305 (1994).

[39] P. S. Wesson, Phys. Lett. B 276, 299 (1992).

[40] H. Liu and P. S. Wesson, J. Math. Phys. 33, 3888 (1992).

[41] P. S. Wesson and J. Ponce de Leon, Class. Quant. Grav. 11, 1341 (1994).

[42] H. Liu and P. S. Wesson, Class. Quant. Grav. 13, 2311 (1996).

[43] A. Billyard and P. S. Wesson, Phys. Rev. D 53731 (1996).

[44] P. H. Lim and P. S. Wesson, Astrophys. J. 397, L91 (1992).

[45] D. Kalligas, P. S. Wesson and C. W. F. Everitt, Astrophys. J. 439, 548 (1995).

[46] P. H. Lim, J. M. Overduin and P. S. Wesson, J. Math. Phys. 36, 6907 (1995).

[47] P. S. Wesson, H. Liu and P. H. Lim, Phys. Lett. B 298, 69 (1993).

[48] H. Liu, P. S. Wesson and J. Ponce de Leon, J. Math. Phys. 34, 4070 (1993).

[49] H. Liu and P. S. Wesson, Phys. Lett. B 381, 420 (1996).

[50] K. Gödel, Rev. Mod. Phys. 21, 447 (1949).

[51] M. M. Som and A. K. Raychaudhuri, Proc. Roy. Soc. London A 304, 81 (1968).

[52] A. Banerjee and S. Banerji, J. Phys. A 1, 188 (1968).

[53] F. Bampi and C. Zordan, Gen. Rel. Grav. 9, 393 (1978). 
[54] M. Novello and M. J. Rebouças, Phys. Rev. D 19, 2850 (1979).

[55] M. J. Rebouças, Phys. Lett. A, 70, 161 (1979).

[56] A. K. Raychaudhuri and S. N. G. Thakurta, Phys. Rev. D 22, 802 (1980).

[57] S. K. Chakraborty, Gen. Rel. Grav. 12, 925 (1980).

[58] M. J. Rebouças and J. Tiomno, Phys. Rev. D 28, 1251 (1983).

[59] A. F. F. Teixeira, M. J. Rebouças and J. E. Åman, Phys. Rev. D 32, 3309 (1985).

[60] M. J. Rebouças and A. F. F. Teixeira, Phys. Rev. D 34, 2985 (1986).

[61] M. J. Rebouças, J. E. Åman and A. F. F. Teixeira, J. Math. Phys. 27, 1370 (1986).

[62] M. J. Rebouças and J. E. Åman, J. Math. Phys. 28, 888 (1987).

[63] F. M. Paiva, M. J. Rebouças and A. F. F. Teixeira, Phys. Lett. A 126, 168 (1987).

[64] K. Dunn, Gen. Rel. Grav. 21, 137 (1989).

[65] R. X. Saibatalov, Gen. Relativ. Grav. 7, 697 (1995).

[66] A. Krasiński, J. Math. Phys. 39, 2148 (1998). This reference containsf a brief overview of the literature on rotating models in general relativity.

[67] E. P. V. Vaidya, M. L. Bedran and M. M. Som, Prog. Theor. Phys. 72, 857 (1984).

[68] L. L. Smalley, Phys. Rev. D 32, 3124 (1985).

[69] J. D. Oliveira, A. F. F. Teixeira and J. Tiomno, Phys. Rev. D 34, 3661 (1986).

[70] L. L. Smalley, Phys. Lett. A 113, 463 (1986).

[71] W. M. Silva-Jr., J. Math. Phys. 32, 3223 (1991).

[72] A. J. Accioly and A. T. Gonçalves, J. Math. Phys. 28, 1547 (1987).

[73] A. J. Accioly and G. E. A. Matsas, Phys. Rev. D 38, 1083 (1988).

[74] T. Singh and A. K. Agrawal, Fortschr. Phys. 42, 71 (1994). 
[75] J. E. Åman, J. B. Fonseca-Neto, M. A. H. MacCallum and M. J. Rebouças, Class. Quant. Grav. 15, 1089 (1998).

[76] M. J. Rebouças and A. F. F. Teixeira, Riemannian Space-times of Gödel Type in Five Dimensions, to appear in J. Math. Phys. (1997).

[77] J. E. Åman, "Manual for CLAssi: Classification Programs for Geometries in General Relativity", Institute of Theoretical Physics Technical Report, University of Stockholm (1987). Third provisional edition. Distributed with the SHEEP sources.

[78] M. A. H. MacCallum and J. E. F. Skea, "Sheep: A Computer Algebra System for General Relativity", in Algebraic Computing in General Relativity, Lecture Notes from the First Brazilian School on Computer Algebra, Vol. II, edited by M. J. Rebouças and W. L. Roque. Oxford U. P., Oxford (1994). 